In Crescendo, 2019; 10(1): 13-34

Fecha de recepción: 22 de enero del 2019

Fecha de aceptación: 22 de marzo del 2019

\title{
LA IMPORTANCIA DE LA GESTIÓN CURRICULAR UNIVERSITARIA EN PROGRAMAS A DISTANCIA, ESTUDIO INSTITUCIÓN DE EDUCACIÓN SUPERIOR SURAMERICANA
}

\author{
THE IMPORTANCE OF UNIVERSITY CURRICULAR MANAGEMENT \\ IN REMOTE PROGRAMS, STUDY INSTITUTION OF \\ SOUTH AMERICAN HIGHER EDUCATION
}

\author{
Myriam Fabiola Benavidez-Torres ${ }^{1}$, \\ Lylliana Vásquez-Benitez ${ }^{2}$
}

\section{RESUMEN}

La investigación realizada se basa en la gestión curricular universitaria al interior del programa de Administración en Salud Ocupacional modalidad distancia, parte del análisis del sistema de gestión curricular del programa, identificando cuál es el papel de su gestión en la Facultad de Ciencias Empresariales de la corporación Universitaria Minuto de dios rectoría Virtual y a Distancia Colombia, el estudio es cualitativo de corte descriptivo y transversal, el aporte de la investigación se dio en el mejoramiento y entendimiento de los procesos curriculares que fortalecen la gestión curricular en los programas a distancia, permitiendo identificar los cuellos de botella y acciones de mejora que fortalezcan el quehacer pedagógico y de práctica docente, se trabajó con docentes, estudiantes, currículo, los resultados denotan el desconocimiento parcial tanto del modelo educativo institucional, como del modelo curricular y la carencia de un modelo de gestión curricular apropiado para el modelo de educación a distancia.

1 Ingeniero Química UNAL Colombia, Especialista en ciencia y tecnología de alimentaros UNAL Colombia, Magíster Gestión y auditorías ambientales Universidad Internacional Iberoamericana, Magíster Educación Corporación Universitaria Minuto De Dios Colombia, Directora Curricular Corporación Universitaria Minuto de Dios- UNIMINUTO Virtual y a Distancia, email myfabeto@gmail.com, ORCID https://orcid.org/0000-0003-0758-710X.

2 Docente maestría en Educación Corporación Universitaria Minuto De Dios Colombia, UNIMINUTOVirtual y a Distancia.lvasqu16@uniminuto.edu.co 
PALABRAS ClAVE: Gestión académica, Gestión educativa, Gestión escolar, Educación a distancia, Enseñanza y formación. ${ }^{3}$

\section{ABSTRACT}

The research carried out is based on the university curricular management within the Occupational Health Administration program distance modality, part of the analysis of the curriculum management system of the program, identifying what is the role of its Management in the Faculty of Business Sciences of the University Corporation minute of God Virtual rectory and distance Colombia, the study is qualitative descriptive and transversal cut, the contribution of the research was given in the improvement and understanding Of the curricular processes that strengthen the curricular management in the distance programs, allowing to identify the bottlenecks and improvement actions that strengthen the pedagogical task and of teaching practice, worked with teachers, students, Curriculum, the results denote the partial ignorance of both the institutional educational model and the curricular model and the lack of a model of curricular management appropriate for the model of distance education.

KEY WORDS: Academic management, educational management, school management, distance education, education and training.

\section{INTRODUCCIÓN}

La gestión curricular como un proceso académico se convierte en la herramienta de toma de decisiones curriculares relevante en los programas de modalidad a distancia, el estudio se realizó en la facultad de Ciencias Empresariales distancia de la Corporación Universitaria Minuto de Dios - Colombia, programa de Administración en Salud Ocupacional, se indagó sobre la existencia de un modelo de gestión curricular, un estudio de los investigadores Maldonado, E., \& Seehusen, V. (2018), relacionan que la investigación sobre la gestión curricular presenta insumos importantes como el proceso de rediseño curricular en los programas de negocios, donde como ejercicio se identifican ejes de trabajo nuevos como la economía de Marketing-Management y Marketing-Management, convirtiéndose en un aspecto de trabajo asociado a la actualización curricular deseada en las universidades que logra que el estudiante tenga mayor campo de acción laboral.

3 Tesauro de la UNESCO es una lista controlada y estructurada de términos para el análisis temático y la búsqueda de documentos y publicaciones en los campos de la educación, cultura, ciencias naturales, ciencias sociales y humanas, comunicación e información. Continuamente ampliada y actualizada, su terminología multidisciplinaria. 
Según Alvarado-Herrera, S. S., González-Sandoval, G. E., \& PaniaguaCortés, Y. (2018), la gestión curricular permite el planteamiento de estrategias de mejora en la formación docente, se logra el entendimiento en el marco de las tendencias mundiales, estos espacios académicos de acreditación, permiten que la gestión académica y liderazgo compartido, enfaticen en un modelo de reconstrucción conceptual que depende del nivel de formación, como de su estructura y el desarrollo en el aula.

Para acercarnos al fenómeno estudiado se identificaron los actores y cuáles son los componentes, no obstante, Rodríguez-Castro, M. (2018), aporta al concepto de la gestión curricular un argumento interesante que depende de los ajustes de la tecnología y el uso de diseños instruccionales asistidos por computadora, que permiten que cada área del saber tenga la mejor personalización, si este diseño es permitente y consistente se logra un incremento en la aplicación en los estudiantes, sin embargo, el nivel de complejidad se da en el lenguaje en el cual las habilidades o técnicas son definidas.

\section{MODELOS DE GESTIÓN CURRICULAR}

Para Jiménez, N. E. L. (2016), se reconoce la gestión curricular, como la construcción de espacios de libertad conceptual y teórica, pero con autonomía institucional, la cual determina el derrotero desde el modelo pedagógico, pretende dar respuesta al iqué se debe enseñar? y icómo se lograría el aprendizaje?, permitiendo la fusión de modelos cognitivos, que identifiquen el objeto de saber, presentando una percepción de la realidad del programa a diseñar, permitiendo la trascendencia cultural y la prevalencia de su contexto desde el área de conocimiento aplicado, la gestión curricular es un constructo multidimensional, determinado por un gran número de variables (inteligencia, motivación, personalidad, etc.) y en el que influyen numerosos factores personales, familiares o escolares, entre otros" según (Adell, 2006, p. 17).

Desde lo anterior se plantea la integralidad del currículo, como el todo, que no solo es un documento, si no que establece escenarios y modelos de aplicación, recursos obligatorios que generen un aporte, para Rodríguez, R. R. (2017), reconoce como la investigación y su relación con el diseño curricular llevan a determinar la praxis que tiene correspondencia cuando un programa académico es ofertado, es allí donde se genera el estado de la cuestión del cómo se pueden formar profesionales que respondan a las necesidades sociales. 


\section{EL CURRÍCULO COMO FUENTE DE APRENDIZAJE}

Una experiencia importante de desarrollo de ambientes de aprendizaje, según Montiel, E., \& Castillo, A. (2016), son las experiencias de aula, el ejercicio se dio en Guatemala donde se logra un diseño con la apropiación de la realidad que permite la flexibilidad y el enriquecimiento de los contextos educativos, aumentando desde la cotidianidad el incremento de los recursos aplicados.

Para Hunter, L., \& Lean, J. (2018), la experiencia realizada en Kenia y Tanzania, el currículo se piensa y se aprende desde la mirada del emprendimiento social que se particulariza con las necesidades de formación de las problemáticas del contexto, este aprendizaje se lleva a las aulas de colegios y universidades donde podría interpretarse que lo anterior plantea un currículo no tradicional mediado por la enseñanza de su heurística y la evaluación, Peralta, D. A. S., \& Aguja, F. A. P. (2015), la evaluación como infraestructura sistemática del currículo, esta estructura con la que se ha venido trabajando desde el siglo pasado en Latinoamérica no permite su dinamismo social, y que actualmente se replantea mediante la observación de otros modelos que invitan a una transformación que no anquilósale los diseños y formas de gestionar la actualización curricular.

Al respecto Montoya Delgadillo, J. L. (2016), establece que el papel de los modelos por competencias, donde el currículo permite que su comprensión de la realidad transcienda de las aulas universitarias, no solo en las formas de desarrollo, ni también solo de referencia, ni se reconozca como el plan de estudios y que su mirada se da desde la experiencia docente, el currículo entendido de esta forma, termina por ser lo más importante para la gran mayoría de instituciones educativas.

\section{EDUCACIÓN Y LA GESTIÓN CURRICULAR}

Se define la gestión curricular como un conjunto de procesos que están orientados a un fin formativo, a facilitar un aprendizaje determinado, Nicoletti, J. A. (2016), la define como aquel acto educativo que se transforma en la construcción de escenarios apropiados para el discernimiento y la fundamentación, permitiendo el diálogo y la generación de saberes, el Instituto Tecnológico de Monterrey (2010), lo define como una técnica educativa que "es el procedimiento lógico y con fundamento psicológico destinado a orientar el aprendizaje del alumno". 


\section{DIDÁCTICA PARA LOS MODELOS CURRICULARES}

Para Velasco y Mosquera (2008), la didáctica de los modelos curriculares refiere al conjunto de procedimientos, apoyados en técnicas de enseñanza, que tienen por objeto llevar a buen término la acción pedagógica del docente; es decir que los docentes deben conocer procedimientos didácticos que les permitan desarrollar de la mejor forma posible la transferencia de conocimiento, es así como se llega a delimitar los métodos a utilizar con cada estudiante según el año de escolarización, Ord, J. (2016), el currículo es un proceso que cuenta con principios y genera un desarrollo, que para Stenhouse (2003), muchas veces es algo directamente orientador para los docentes, de tal forma que le da un orden y una jerarquía a las materias planteadas, establece la relación entre las percepciones de la intención y la realidad.

\section{DEFINICIÓN DE CURRÍCULO}

Según Goodson (1991), en temas referentes a la escolarización, el currículo es una palabra clave debido a que se encierra el desarrollo de diversos aspectos que conciernen a los procesos educativos, en donde se desenvuelven intereses, relaciones, exámenes y análisis. Los principales conflictos curriculares se enmarcan en una evidencia de tipo palpable y documental referente a las razones y los objetivos de la escolarización, es por esta razón que se encuentra importante generar un área del conocimiento que permita dar respuesta a los conflictos curriculares, desde las que se orienten los procesos de los estudiantes para profundizar sobre un área determinada.

Así también se definen formas para la evaluación y práctica del currículo; así mismo se empiezan a engranar de una u otra forma los diversos procesos de tipo administrativo y hasta financiero en torno a los currículos en donde cuestionan niveles, proceso, valores, donde diversos autores a lo largo del tiempo han desarrollado diferentes conceptos sobre currículo, entre los cuales se encuentra Jackson (1968); Green (1971); Young (1977), citados por Goodson (1991), entre las principales características definidas por los autores mencionados, se encuentra la realización proactiva e interactiva del currículo, lo que permite una comprensión de la distinción entre los diferentes grados de este, evidenciando una estructura de conocimiento que permite que la persona aprenda una fundamentación real. 


\section{EVALUACIÓN DE NECESIDADES}

Para poder diseñar el programa de un proyecto formativo es necesario generar el diseño de dicho proyecto, en donde según Zabalza (1997) "la educación ha supuesto un importante avance de cara a dotar de mejor compromiso y siendo operativo a las decisiones formativas alejándolas de planteamientos voluntaristas, indefinidos y difícilmente sometidos a crítica y evaluación" (p. 62), continuando con la definición de necesidad es vital la incorporación de diversos componentes a nivel integral dentro de los currículos, generando un desarrollo de tipo intelectual, afectivo, social, entre otros, que generen una identificación de diversos aspectos en el desarrollo de una persona, también es necesario observar las diferencias individualizadoras de cada sujeto que le permiten desarrollar la personalidad, y la libertad de pensamientos y criterios; a la vez un crecimiento a nivel personal y académico.

\section{LA GESTIÓN CURRICULAR EN COLOMBIA}

La gestión curricular en Colombia tiene como punto de referencia inicial la organización del sistema educativo, cuyo principio rector se encuentra enmarcado en la Constitución Política de Colombia (1991), la ley General de Educación Colombiana (Ley 115 de 1994) y el decreto 1075 de mayo de 2015, normatividad referente para el desarrollo de esta investigación, la Constitución Política en sus artículos 45 y 67 de 1991 de Colombia define la educación como un servicio público, al cual tienen derecho todos los ciudadanos y ciudadanas del país sin distingo de sexo, raza, ideología política, nivel socioeconómico o credo religioso y como un servicio público que tiene una función social. Con la educación se busca el acceso al conocimiento, a la ciencia, a la técnica y a los demás bienes y valores de la cultura. Le corresponde al estado velar por la calidad de la educación y garantizar el acceso y permanencia de los menores en el sistema educativo.

Ley General de Educación (Ley 115 de 1994) Artículos 5 y 78 Establece la educación formal, entendida como aquella que se "imparte en establecimientos educativos aprobados oficialmente y se desarrolla en una secuencia regular de ciclos lectivos, con sujeción a pautas curriculares progresivas, y conducente a grados y títulos". La misma ley puntualiza que la educación se organiza en tres niveles: El preescolar, La educación básica y la educación media que tiene el carácter de académica o técnica. A su término se obtiene el título de bachiller que habilita al educando para ingresar a la educación superior y al trabajo.

Esta misma ley define las áreas obligatorias y fundamentales, dentro de las cuales se contempla la enseñanza de la lengua castellana, como lengua materna. 
El Artículo $5^{\circ}$ plantea los fines de la educación. En los numerales 5, 6, 7 y 9, se hace explícita la necesidad de formar un pensamiento crítico, reflexivo y analítico, propósitos fundamentales del presente proyecto.

\section{NUEVAS PROYECCIONES EN EL CURRÍCULO}

Según Lafrancesco (2005), se han desarrollado nuevos fundamentos para la transformación curricular, en donde se debe considerar para un cambio educativo de calidad, que permita contribuir a los procesos de cualificación de la misma y generar una forma de pensar en la educación acorde a las exigencias particulares y socioculturales que deben asumir las instituciones educativas del país. Es así como dentro de los puntos comunes de modificación que se observan están: objetivos, selección y organización de contenidos, selección y organización de experiencias de aprendizaje y evaluación, a la vez que se examinan algunos de los contextos esenciales para los procesos de dicha transformación: el conocimiento científico y tecnológico, desarrollo humano, procesos formativos integrales, la respuesta a las necesidades sociales y culturales, los nuevos aportes pedagógicos, tendencias actuales de la administración educativa y globalización, exigencias en el campo de educación y de la gestión curricular.

\section{LA GESTIÓN CURRICULAR EN LA EDUCACIÓN SUPERIOR}

Desde el año 2008 el Ministerio de Educación Nacional de Colombia, se ha orientado a formular diversas competencias genéricas o transversales a todos los núcleos de formación en educación superior que posibiliten un monitoreo de la calidad de la educación superior en el país y que puedan constituirse en el elemento articulador de todos los niveles educativos: inicial, básica, media y superior. Entre los Lineamientos para la formación por competencias en educación superior se encuentran:

1. Competencias abstractas del pensamiento: razonamiento crítico, entendimiento interpersonal, pensamiento creativo, razonamiento analítico y solución de problemas.

2. Conocimientos y competencias prácticas necesarias para el despliegue de las competencias abstractas: conocimiento del entorno, comunicación, trabajo en equipo, alfabetización cuantitativa, manejo de información, comunicación en inglés y TICS.

3. Dinamizadores para el desarrollo de las competencias genéricas: Saber aprender y re contextualizar. 


\section{MODELOS DE GESTIÓN CURRICULAR}

DeVries e Ibarra (2004), mencionan que la gestión es un campo poco explorado en educación superior, por su parte Morantes y Acuña (2013), refieren un estudio según el cual las temáticas de investigación han ido cambiando de la investigación centrada en tecnologías hacia otras áreas como la gerencia y el cambio, "Todos los aspectos relacionados con la gerencia educacional están creciendo en importancia (estrategia, liderazgo, aseguramiento de la calidad, etc.), sin embargo Pardasani, M. (2018), reconoce que se puede trabajar con modelos curriculares innovadores no solo en lo académico sino en todos los aspectos mencionados que hacen parte de la gestión curricular de alguna manera.

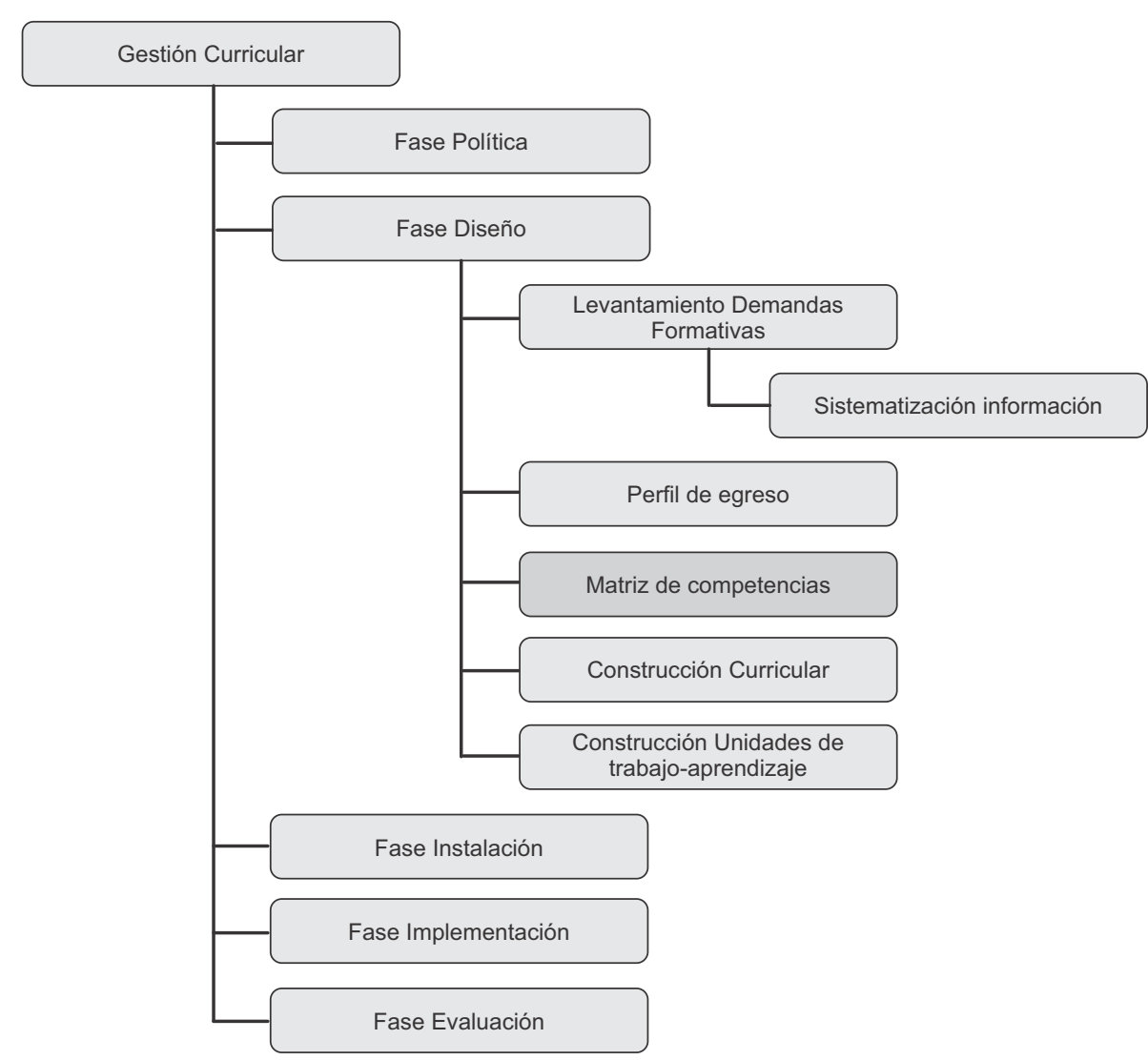

Figura 1. Gestión Curricular; Fuente: Tobón, (2008). 
De otra parte, Duque (2009), define un modelo de gestión como la forma de organizar y combinar los recursos para cumplir con los objetivos. Corresponde también con políticas, proceso, procedimientos y pautas definidas para mejorar el desempeño de la organización. Desde este punto de vista, es de esperarse que la gestión curricular esté orientada por procedimientos establecidos desde la misma institución propendiendo por la organización, el aprovechamiento de los recursos disponibles y fundamentalmente buscando una mejora continua de los programas académicos.

A partir de lo anterior, podemos identificar que existen otros modelos de gestión que presentan diferentes estructuras, así mismo generar otros procesos que permiten a la entidad profundizar en la gestión del currículo en las instituciones de educación superior. En consecuencia, de lo anterior, se presenta el modelo diseñado por Sánchez, (2016).

-Abierto, flexible y dinámico

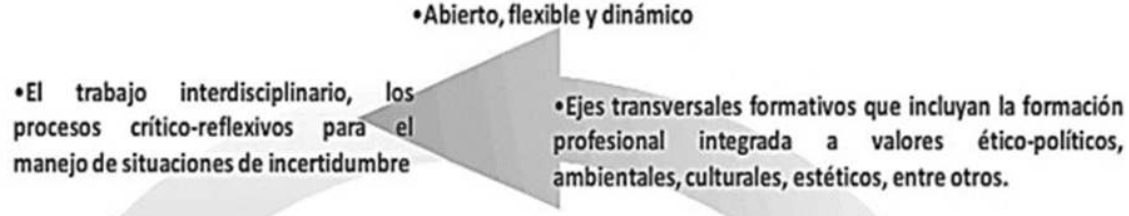

- Los proyectos como estrategia de aprendizaje y evaluación

- La formación integral mediante la organización en unidades integrales de formación

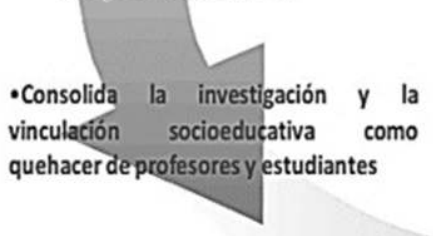

-Privilegia el aprendizaje en vez de la enseñanza

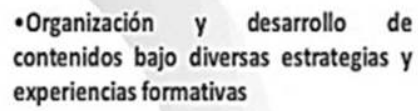

EL DISEÑO CURRICULAR

- Conocimientos y saberes pertinentes en sus alcances nacional, regional y mundial

-Ejercicio de la democracia participativa, el diálogo y la comunicación horizontal
- Programas con diseños curriculares armonizados que eviten la heterogeneidad y la frondosidad curricular

Figura 2.Diseño curricular de los programas nacionales; Fuente: Sánchez (2016). 
Es posible definir un modelo de gestión curricular como un ejercicio en el que se ven involucrados diferentes actores y elementos del ejercicio del contexto educativo, participando de manera organizada, en un ejercicio que tiene como finalidad principal la actualización, para Vincent, S., \& Focht, W. (2011), existen elementos del currículo que trabajan de manera interdisciplinar y permiten la definición de su perfil dando una identidad apropiada con su diseño, identificando los términos académicos y la mejora en términos de procesos, "la educación como un acontecimiento social que ocurre cada vez que hay un encuentro humano, en tanto que las ciencias educacionales son reflexiones para comprender y mejorar el fenómeno de la enseñanza-aprendizaje" (Vílchez 2004).

Desde el concepto de currículo, es posible identificar tres perspectivas: 1) Como Construcción social, es decir como puente entre la sociedad y la escuela, Montes, C. S. (2015), currículo por objetivos y competencias 2) Como proyecto educativo; en el que se representan los contenidos y las secuencias y 3) Como una unidad en la que convergen diferentes prácticas que no son solo de carácter pedagógico, sino que se consideran las interacciones y relaciones con el contexto. (Sacristán, 1998). También se presenta como la innovación social como aporte a la educación por esto Aguja, F. A. P., \& Quintero, H. J. G. (2017), en la espiral de la innovación la consideran el valor de mayor aporte, por su parte, la gestión curricular es "comprendida como un conjunto de procesos, decisiones y prácticas para estimular, dinamizar, desarrollar y reflexionar en torno al currículo y su buena práctica. (Volante, 2015).

\section{MÉTODO}

Para desarrollar esta investigación se aplicó una metodología cualitativa, descriptiva, y transversal según Hernández Sampieri, R., Fernández Collado, C., \& Baptista Lucio, P. (2010), se busca responder, explicar y justificar lo evidenciado a través de las descripciones o explicaciones, resultantes de la aplicación de una ficha de observación de procesos organizacionales, y que su vez constituye el instrumento base de la investigación; se aplicaron entrevistas semiestructuradas, y una ficha de caracterización de procesos que permite observar paso a paso el proceso que se lleva actualmente al interior del programa para la actualización y gestión curricular.

Este recoge información y datos sometidos a técnicas hermenéuticas que permiten, no solo explicar fenómenos sociales, sino también predecir posibles 
Tabla 1

SELECCIÓN POBLACIONAL

\begin{tabular}{lc}
\hline Cargo & Número-cantidad \\
\hline Decano & 1 \\
Directores de programa & 1 \\
Profesores tiempo completo & 12 \\
Profesores medio tiempo & 34 \\
Secretaria académica & 1 \\
\hline Total & 49
\end{tabular}

Fuente: elaboración propia.

Tabla 2

MATRIZ DE CATEGORÍAS

\begin{tabular}{llc}
\hline Categoría & Objeto de la categoría & Valor de la categoría \\
\hline $\begin{array}{l}\text { Composición } \\
\text { curricular }\end{array}$ & $\begin{array}{l}\text { Identificar la composición interna de } \\
\text { sistema de gestión curricular que } \\
\text { presenta el sistema universitario. }\end{array}$ & CC_01 \\
\hline $\begin{array}{l}\text { Cohesión y } \\
\text { Coherencia } \\
\text { curricular }\end{array}$ & $\begin{array}{l}\text { Comprender la articulación y entrelace } \\
\text { de los componentes del modelo }\end{array}$ & COCH_02 \\
\hline $\begin{array}{l}\text { Proyección del } \\
\text { currículo }\end{array}$ & $\begin{array}{l}\text { Observar el comportamiento de } \\
\text { currículo aplicado en el sistema }\end{array}$ & \\
& universitario y sus posibles mejoras. & PRC_03 \\
\hline
\end{tabular}

Fuente: elaboración propia.

escenarios futuros; en este caso, lograr con el estudio el diagnóstico y propuesta de un sistema de gestión curricular que permita una actualización permanente y responda a las necesidades de los programas en el cumplimento de los objetivos de formación respectivos, la población (ver tabla 1), directivos, docentes, administrativos, el muestreo se dio por conveniencia, Hernández Sampieri, R., Fernández Collado, C., \& Baptista Lucio, P. (2010), determina que se caracteriza por 
un esfuerzo deliberado de obtener muestras "representativas" el grupo poblacional fue elegido a conveniencia donde se estudió a un conjunto específico de personas que se encuentran de un grupo más grande, los profesores seleccionados para el desarrollo de este estudio, participan en el comité curricular del programa, y de allí, el interés de seleccionarlos de manera conveniente para la obtención de información veraz y rigurosa para el análisis (mecanismo de selección de los participantes).

Las categorías que se analizarán en la presente investigación corresponden a los aspectos más relevantes en la gestión curricular en el programa Administración en Salud Ocupacional de la Facultad de Ciencias Empresariales; tales son composición curricular; cohesión y coherencia curricular; proyección del currículo.

\section{RESULTADOS}

Síntesis inicial: según los resultados e información recolectada de los encuestados, no existen una metodología identificada para la gestión curricular; de acuerdo con lo referido por los colaboradores de la institución, vinculados en procesos académicos del programa que se analiza, entrevistado 1: "desde hace poco se ha venido hablando sobre gestión curricular y algunos de los profesores y directivos han identificado que la forma de gestión curricular que se aplica es la sugerida por las instancias académicas superiores", la cual pretende que los ejercicios de reflexión curricular surtan un recorrido por "comité de programa, comité curricular único de programa y comité nacional de centros tutoriales o sedes”.

\section{Tabla 3}

\section{PROCESOS DE INVESTIGACIÓN}

aplicado según 1: gestión curricular; proceso 2: construcción curricular; proceso 3: análisis curricular; proceso 4: evaluación curricular

\begin{tabular}{|c|c|}
\hline \multicolumn{1}{|c|}{ Planear } & \multicolumn{1}{c|}{ Hacer } \\
\hline ¿Cómo lo planean? & ¿Cómo lo desarrollan? \\
\hline Verificar & \multicolumn{1}{|c|}{ Actuar } \\
\hline ¿Qué procesos utilizan para verificarlo? & ¿Cómo lo aplican? \\
\hline
\end{tabular}

Fuente: elaboración propia. 
Tabla 4

RESULTADOS DE LA ENTREVISTA SEMIESTRUCTURADA

\begin{tabular}{|c|c|c|}
\hline Categoría & Código & Interpretación \\
\hline $\begin{array}{l}\text { Concepto de } \\
\text { Gestión } \\
\text { Curricular }\end{array}$ & \multirow[t]{3}{*}{ CC_01 } & $\begin{array}{l}\text { De acuerdo con las respuestas de los docentes, el } \\
\text { concepto de la gestión curricular no es claro, se analiza } \\
\text { que en su mayoría guardan elementos de relación, } \\
\text { comparación y similitudes. }\end{array}$ \\
\hline Pregunta 1 & & $\begin{array}{l}\text { El concepto se configura con elementos claves que } \\
\text { dejan ver cierta claridad en el conocimiento del pro- } \\
\text { ceso de gestión curricular por su conceptualización. }\end{array}$ \\
\hline & & $\begin{array}{l}\text { Expresan que la gestión curricular es un proceso o } \\
\text { procedimiento que requiere un orden, un protocolo } \\
\text { establecido. Corresponde con poner en práctica los } \\
\text { conocimientos, habilidades y reflexiones concretas } \\
\text { para comprender y transformar el proyecto curricular } \\
\text { de un programa académico de educación superior en } \\
\text { el contexto de la Institución de Educación Superior } \\
\text { dando respuesta a las necesidades del sector produc- } \\
\text { tivo y del contexto social. }\end{array}$ \\
\hline
\end{tabular}

\section{Proceso de \\ Gestión \\ Curricular \\ CC_01}

Preguntas 3, 4 y

9
Los docentes en sus respuestas exponen significativas apreciaciones sobre las actividades que se realizan y que corresponden o hacen parte del proceso de gestión curricular. Señalan aspectos del proceso que se relacionan con eventos de construcción curricular, análisis y evaluación curricular. Por su parte, los directivos refieren diferentes respuestas sobre el proceso que se adelanta en el programa y mencionan aspectos resultantes de proceso de autoevaluación y acciones de mejoramiento continuo. No se define o infiere a través de las respuestas y las observaciones realizadas un proceso de gestión curricular como tal.

En relación con el método HPVA, no se aprecian respuestas importantes.

Aun así, se reconoce en varias respuestas que tanto profesores como directivos indican la necesidad de la definición de un proceso y orientaciones claras, su implementación, en la búsqueda de propuestas académicas y curriculares pertinentes. 
Tabla 4 (Cont.)

\begin{tabular}{|c|c|c|}
\hline Categoría & Código & Interpretación \\
\hline $\begin{array}{l}\text { Modelo de } \\
\text { Gestión } \\
\text { Curricular } \\
\text { Preguntas } 5,6 \text { y } \\
8\end{array}$ & COCH_O2 & $\begin{array}{l}\text { Se manifiesta gran interés de parte de docentes y } \\
\text { directivos por el modelo de gestión curricular y la } \\
\text { necesidad de suadecuada implementación. } \\
\text { Sin embargo, los docentes no evidencian conoci- } \\
\text { miento por los instrumentos de gestión que la institu- } \\
\text { ción tiene definidos a través del Proyecto Educativo } \\
\text { Institucional, tales como modelo educativo, modelo } \\
\text { de gestión y modelo curricular. } \\
\text { En las respuestas se observa uso del término modelo } \\
\text { educativo o modelo curricular como el lineamiento } \\
\text { para la gestión curricular. Además de lo anterior, } \\
\text { refieren las fases del modelo educativo con enfoque } \\
\text { praxeológico como las fases de un modelo de gestión } \\
\text { curricular al interior del programa. } \\
\text { Por su parte directivos del programa y la facultad, } \\
\text { aunque no define un modelo de gestión curricular, lo } \\
\text { relacionan con actualización, cambo y reforma, ade- } \\
\text { más de articulación con el contexto y particularmente } \\
\text { con el sector productivo. } \\
\text { Refieren como modelo en algunos casos el ejercicio que } \\
\text { se realiza a través de los comités curriculares, aclarando } \\
\text { que existen lineamentos generales para su conforma- } \\
\text { ción y para las reuniones o encuentros, pero no se } \\
\text { cuenta con protocolos, metodología o procedimientos } \\
\text { definidos para su operación; apenas si hay una mínima } \\
\text { definición de funciones amplias que deben desarrollar. }\end{array}$ \\
\hline
\end{tabular}

Articulación y
pertinencia de
documentos
institucionales
con modelo de
gestión
curricular

Preguntas 2, $7 \mathrm{y}$

9
Si bien los docentes de la muestra seleccionada aleatoriamente señalan la existencia de documentos institucionales tales como: el modelo educativo praxeológico, el sistema de investigaciones, el plan de currículo, entre otros, expresan que estos no tienen las suficientes claridades sobre las fases de un modelo de gestión curricular. Los documentos institucionales no presentan la metodología pertinente, que defina los criterios institucionales y normativos, que permita realizar procesos de construcción, análisis y evaluación curricular propios de la gestión curricular y que faciliten procesos sistemáticos y permanentes para actualización de propuestas curriculares de programas académicos. 
Para el proceso 1: Gestión Curricular; los profesores manifiestan que la Planeación curricular tiene lugar desde el momento en el cual se formula el plan de estudios o ruta sugerida para solicitar el registro calificado del programa, teniendo en cuenta las condiciones de los aspectos curriculares, luego pasa por la preparación de cada curso propuesto en el plan de estudios; estos cuentan con una propuesta académica de micro currículo, un diseño metodológico de guías de trabajo y diseño instruccional y aulas virtuales. El trabajo en cada curso está dispuesto para 16 semanas y cada uno de ellos es definido en términos de créditos académicos de acuerdo con la reglamentación legal vigente del Ministerio de Educación Nacional MEN; las horas de trabajo directo y con acompañamiento son definidas en la ruta sugerida. La planeación se realiza de manera anual para las actividades de operación del programa, considerando los siguientes criterios; organización de tiempo, definición de equipo de trabajo, preparación de planes de trabajo, asignación de roles y funciones. (versión simplista).

Los profesores del programa consideran importante identificar un experto disciplinar con experiencia en el ejercicio de actualización y desarrollo curricular con el fin de que determine los avances o los ajustes requeridos en el currículo propuesto para cada asignatura. (Versión holística) En relación con la forma cómo desarrollan la gestión curricular, las respuestas refieren que el plan de estudios se desarrolla por medio de la operación de este a través del periodo académico, tratando de llevar una secuencia lógica sugerida y discusiones académicas sustentadas en comité curricular; el estudiante toma los cursos establecidos en la ruta sugerida o plan de estudios.

Eje 2: Construcción Curricular; tiene lugar a través de la reflexión, análisis y revisión epistemológica, de tendencias y necesidades del sector laboral, teniendo como referencia los cambios en la normatividad y la necesidad del programa; de acuerdo con esto se revisa la pertinencia del plan de estudios a la luz de la reglamentación legal vigente para el campo disciplinar. Una vez se ha hecho este trabajo se procede a establecer la propuesta y se trabaja desde planeación curricular para diseñar, ajustar o rediseñar el plan de estudios y la ruta sugerida junto con el comité curricular del programa.

La verificación de la construcción curricular se realiza teniendo como referencia los cambios en la normatividad y la necesidad del programa, por medio del comité curricular, y a la luz de los planes de mejoramiento resultantes del proceso de autoevaluación, de ser necesario, el proceso cuenta con pares amigos evalua- 
dores, bien sea para revisión de contenidos de cursos, del plan de estudios o del proyecto curricular.

Eje 3: Análisis Curricular; Este proceso no es claro entre los profesores vinculados al programa. más, sin embargo, la evaluación curricular se planea y se realiza permanentemente a través de jornadas académicas en cada periodo desde el momento en el cual se establece la relación entre propósitos de formación, perfiles y competencias. Esto se puede ver a través de las evaluaciones de los estudiantes al interior de cada curso, los proyectos de grado y la gestión de la estructura de evaluación de cada espacio académico.

Eje 4: Evaluación Curricular; No se cuenta con políticas claras desde la vicerrectoría académica de sede o del sistema, por lo que la evaluación se realiza a través del desarrollo de las materias, con retroalimentación de contenidos y estrategias por parte de los profesores. La verificación se materializa con los resultados de los proyectos de grado, los trabajos que tienen lugar al interior de los semilleros de investigación, los resultados de las pruebas Saber-Pro. Para verificar, se observan, a manera de comparación, los contenidos y plan de estudios con los de otras instituciones que ofertan el mismo programa con normatividad vigente. No hay claridad en este proceso; se aplica en los procesos de autoevaluación principalmente por medio de la puesta en marcha de los planes que el docente tiene para el desarrollo del currículo.

La categoría CC_01 mostró que el común denominador en el programa Administración en Salud Ocupacional es la ausencia de una estructura o modelo de gestión curricular, esto se ha identificado mediante las respuestas por parte de los directivos de la facultad, así mismo, los entrevistados han afirmado que lo más estructurado que han podido apreciar de la gestión curricular son las reuniones de comité curricular.

En la categoría COCH_02, se ha logrado identificar dos opiniones divididas sobre la gestión curricular y un posible modelo existente que carece de bases para considerarse como tal; algunos profesores del programa están de acuerdo, y otros difieren, se hace necesario entonces conocer dichas opiniones en detalle para lograr un análisis imparcial y orientado hacia la objetividad. Algunos profesores y directivos opinan que al no existir un modelo curricular se acoplan a los comités curriculares, en ese sentido, no existirían ningún tipo de característica a falta de este. 
En la categoría COCH_02, las respuestas logradas infieren que se está trabajando para dar respuesta de manera adecuada a los criterios de las empresas y al mundo laboral. Es claro que los profesores apropian la idea de que es necesario que el programa esté alineado con las exigencias del sector productivo en lo que respecta a la formación de profesionales. Aun así, resulta contradictorio el hecho de que el programa se presenta a un proceso de renovación de registro calificado con una modificación importante en aspectos como denominación y estructura curricular.

Para la categoría PRC_03, la información ha permitido observar que algunos profesores no reconocen el proceso de evaluación curricular en el programa; aun así, los profesores que apoyan en el ejercicio de propuesta de modificación y actualización curricular con modificación y cambio de plan, y comentan que usan como guía el documento normativo del MEN. Otros profesores, por su parte refieren que el programa debe tener cambios ya que el sector lo requiere, pero no informan cual es el argumento para esta afirmación. Por último, algún profesor menciona el tema de asignación presupuestal para ejecución de acciones de mejora en el programa, lo que no corresponde directamente con las perspectivas evaluativas para el currículo.

\section{MODELO DE GESTIÓN CURRICULAR EN UNIMINUTO}

De esta manera, con la información base del programa en salud ocupacional, se encuentran modelos de gestión como el que refiere Shirley Grundy, que se identifica como un modelo de gestión curricular crítico, soportado en el trabajo cooperativo, interés emancipador, concepto de libertad, relacionado con el habla, expresión y compresión, con ello se busca la verdad y la justicia, lo anterior, se trae a colación, en consideración a que el modelo de Grundy presenta características que pueden ser reconocidas también en el Sistema de la IES, tal como lo denota el proyecto educativo institucional PEI, que refiere que el modelo educativo se propone desde una perspectiva de pensamiento sistémico, que integra las actividades educativas al contexto marco - político, económico, social, religioso, legal, cultural y tecnológicos, local, regional, nacional, el PEI de la IES también presenta las funciones sustantivas desde la perspectiva del modelo, recordando que su objetivo central es la formación integral; así, en la proyección social el objetivo es el desarrollo de personas y comunidades; en la docencia, además de orientar y acompañar el proceso de enseñanza aprendizaje. Por todo lo anterior, el 
modelo de gestión curricular no permite la articulación de todos los actores que intervienen en este proceso, buscando que todos los programas evidencien de manera permanente coherencia, consistencia y especialmente que faciliten la formación integral los estudiantes, con las competencias que requiere el sector externo y en general, un mundo en constante evolución.

\section{DISCUSIÓN}

En relación con la institución, de acuerdo con los resultados y la revisión documental adelantada, no se evidencia la definición de un modelo de gestión curricular, así como lineamientos, según Torres, J. C. C., Ramos, E. C., López, J. E. L., \& Sabatés, J. C. C. (2017), se debe contar con procedimiento que permita la gestión curricular representando la proyección de los programas académicos y sus actores.

Si la institución cuenta con la definición del comité de currículo de programa, integrantes y funciones, desde 2008 a través del Reglamento orgánico, la Resolución Rectoral 1225 de 2010, que define las funciones del comité de currículo del programa, y más recientemente, en 2017, el Consejo General Académico reglamentó los diferentes comités curriculares únicos que operan en el Sistema Universitario de la IES Donoso-Díaz, S. \& Benavides-Moreno, N. (2018), deben existir buenas prácticas de gestión curricular en los componentes directivos, los cuales tendrán la transcendencia especifica al mismo.

La normatividad institucional a este respecto, se implementa de manera adecuada en el programa Administración en Salud Ocupacional, aun cuando se presentan dudas en lo que corresponde a las instancias que avalan propuestas de actualización, modificación o reforma curricular, ya que, en el caso particular del programa Admiración en Salud Ocupacional, está establecido el comité de currículo que preside la Dirección del programa.

\section{CONCLUSIONES}

En el marco de la gestión curricular para el programa Administración en Salud Ocupacional de la Facultad de Ciencias Empresariales de UVD, y acuerdo con los referentes considerados, es preciso hablar del proceso PHVA y cómo este se implementa en el programa, sin embargo, Barraza, C. E. B., \& Gómez, B. P. (2017), Reconoce el modelo de gestión curricular en el marco de las políticas públicas educativas, su gestión permite el desarrollo de la competitividad y la 
innovación, es pertinente mencionar que la gestión curricular es un proceso que debería realizarse en forma permanente como estrategia de mejoramiento continuo y no solo por la coyuntura que corresponde con la normatividad emitida por el MEN en relación con el registro calificado, documento emitido por este ente gubernamental y que faculta a la IES para la oferta académica.

De esta manera, se hace evidente a través de los resultados, que no se cuenta en el programa estudiado con un modelo de gestión curricular implementado que permita una gestión permanente y una evolución constante del programa, en lo que a términos curriculares se refiere. De acuerdo con los resultados de las entrevistas aplicadas, se observa que, aunque se desarrollan muchas actividades tendientes a la evaluación y actualización del programa y los diferentes espacios y actividades que lo componen, no es claro un modelo de gestión curricular o un ejercicio sistemático, que permita tener un seguimiento permanente de los cambios, ni una replicabilidad o trazabilidad del ejercicio. Tampoco se evidencia la existencia de un procedimiento, protocolo o metodología que indique los pasos a seguir, los actores directa e indirectamente implicados, ni las responsabilidades definidas de manera precisa. Aun así, la Dirección del programa es consciente de que es su responsabilidad liderar el ejercicio de reflexión curricular que se concentra en el Comité curricular del programa Administración en Salud Ocupacional de la Facultad de Ciencias Empresariales de UVD.

El programa evidencia cierta desinformación de parte de su cuerpo docente, puesto que solo los directivos y algunos profesores manifiestan estar enterados de los procesos o acciones de gestión curricular que se genera en el programa; esto se traduce en un problema de comunicación que logra una incertidumbre entre lo que se está orientando en las aulas y lo que se plantea desde el proyecto curricular del programa. Herrera, M. A., Fernández, D. C., \& Seguel, R. C. (2018), esta relación a fin de cerrar esta brecha entre lo que se dice y se hace, el ejercicio de gestión curricular debe contar con un canal comunicativo directo que permita el cruce de información, y especialmente de retroalimentación, entre todos los actores involucrados o que en cualquier momento del proceso puedan ser impactados.

Ahora bien, las conclusiones radican esencialmente en la poca evidencia de un modelo de gestión curricular al interior del programa Administración Salud Ocupacional. Con el fin de identificar la existencia o no del modelo, se propusieron algunas categorías de análisis, desde las cuales se puede justificar que la uni- 
versidad requiere prontamente la definición de un modelo o metodología que reconozca cada uno de los programas, su ejercicio académico, los actores que están involucrados y que vaya en concordancia con el modelo educativo y el enfoque de la pedagoga praxeológica, además de estar alineado con el PEI.

En síntesis, en el programa Administración en Salud Ocupacional se identifica una estructura del plan de estudios en coherencia con el modelo curricular establecido a través del PEI. Tanto el plan de estudios de este programa como los de otros programas de la Facultad de Ciencias Empresariales de UVD, evidencian en sus currículos un ejercicio de construcción con visión y justificación integral, en el marco de la normatividad y lineamientos institucionales. Desde la Dirección del programa se orienta el trabajo de gestión curricular buscando dar cumplimiento a los lineamientos generales de la institución en el sentido de generar un espacio de reflexión curricular, programarlo desarrollarlo buscando la participación de los integrantes del mismo según lo establecido en la normativa.

Desde la óptica de los resultados, es importante que este trabajo sirva como base para continuar la investigación sobre el tema de gestión curricular en la IES, particularmente si existen metodologías o aproximaciones a estas, en otros programas, o en otras sedes del sistema de la IES.

\section{REFERENCIAS BIBLIOGRÁFICAS}

Aguja, F. A. P., \& Quintero, H. J. G. (2017). Modelo de gestión del conocimiento solidario, LA RUTA HACIA LA INNOVACIÓN SOCIAL. REVISTA CIENTÍFICA AXIOMA, (16), 49-59.

Alvarado-Herrera, S. S., González-Sandoval, G. E., \& Paniagua-Cortés, Y. (2018). Curricular and Pedagogical Aspects to Be Taken into Account for Curriculum Redesign and Development in a Master's Program in Higher Education. Revista Electrónica Educare, 22 (2), 141-159.

Barraza, C. E. B., \& Gómez, B. P. (2017). Modelo de Gestión en Educación Superior Bajo el Marco de Políticas Públicas. GESTION, COMPETITIVIDAD E INNOVACIÓN, 5 (2).

Cano, M. M. (2009). La investigación escolar: un asunto de enseñanza y aprendizaje en la educación secundaria. Investigación en la escuela, 63-79.

Congreso de la República de Colombia. Ley General de Educación (115/94). Artículo 5 y 78. Bogotá.

Congreso de la República de Colombia. Constitución Política de Colombia 1991. Gaceta Constitucional No. 116. Bogotá D.C. 20 de julio de 1991.

Chuye, Y. (2007). Participación de los actores de la institución educativa en la gestión del cambio. El caso de una escuela pública de Lima (Doctoral dissertation, Tesis para optar el grado de Magíster en Educación con mención en Gestión de la Educación. Lima: Pontificia Universidad Católica del Perú, Escuela de Graduados). 
De Vries, W., \& Colado, E. I. (2004). La gestión de la Universidad. Revista mexicana de investigación educativa, 9(22), 575. Recuperado de:

https://search.proquest.com/openview/fec6d3281565b7af06cd8b05617242ad/1?pqorigsite $=$ gscholar $\&$ cbl $=29479$

Donoso-Díaz, S. \& Benavides-Moreno, N. (2018). Prácticas de gestión de los equipos directivos de escuelas públicas chilenas. Revista Brasileira de Educação, 23, e230013.

Duque Oliva, E. J. (2009). La gestión de la universidad como elemento básico del sistema universitario: una reflexión desde la perspectiva de los stakeholders. INNOVAR. Revista de Ciencias Administrativas y Sociales, 19. http://www.redalyc.org/html/818/81819025003/

Gimeno Sacristán, J. (1998). El currículum: una reflexión sobre la práctica. http://www.sidalc.net/cgi-bin/wxis.exe/?IsisScript =LIBRO.xis\&method=post\& formato $=2 \&$ cantidad $=1$ \&expresion $=\mathrm{mfn}=009116$

Goodson, I. F. (1991). La construcción social del curriculum: posibilidades y ámbitos de investigación de la historia del curriculum. Revista de Educación, (295), 7-37.

Hernández, R, Fernández, C, Baptista, M. (2010). Metodología de la investigación. Quinta Edición. Mc Graw Hill. Perú.

Hernández Sampieri, R., Fernández Collado, C., \& Baptista Lucio, P. (2010). Metodología de la investigación.

Herrera, M. A., Fernández, D. C., \& Seguel, R. C. (2018). Teachers' perception about ICT integration in teaching practices in relation to the normative frameworks for the teaching profession in Chile. Ensaio: Avaliação e Políticas Públicas em Educação, 26(98), 163-184.

Lafrancesco, G. (2005). Nuevos fundamentos para la transformación curricular: a propósito de los estándares. Colombia: Magisterio.

Hunter, L., \& Lean, J. (2018). Entrepreneurial learning-a social context perspective: evidence from Kenya and Tanzania. Journal of Small Business and Enterprise Development.

Jiménez, N. (2016). La construcción curricular: Espacio de libertad y autonomía institucional. Paideia Surcolombiana, (5), 88-99.

Ministerio de Educación Nacional. (1994) Decreto 1860. Artículo 33. Bogotá: Editorial Magisterio.

Ministerio de Educación Guatemala (2013) Situaciones de aprendizaje. Pautas metodológicas para el desarrollo de competencias en el aula. Dirección General de Currículo -DIGECUR.

Ministerio de Educación Nacional. (2013) Propuesta de lineamientos para la formación por competencias en educación superior.

Morantes Higuera, A. E., \& Acuña Corredor, G. A. (2013). Propuesta de modelo de gestión para educación superior a distancia: una aproximación. Zona Próxima, (18). Recuperado de http://www.redalyc.org/html/853/85328617007/

Maldonado, E., \& Seehusen, V. (2018). Data mining student choices: A new approach to business curriculum planning. Journal of Education for Business, 93(5), 196-203.

Montes, C. S. (2015). Competency Curriculum Reform: The Experience in the Preparatorias Federales por Cooperacion. education policy analysis archives, 23, 68 . 
Myriam Fabiola Benavidez-Torres, Lylliana Vásquez-Benítez

Montiel, E., \& Castillo, A. (2016). Diseño de situaciones de aprendizajes. Una experiencia pedagógica en la asignatura química en el nivel de Educación Media General. Educ@ ción en Contexto, 2, 244-260.

Montoya Delgadillo, J. L. (2016). Modelos educativos por competencia y por comprensión en la educación superior.

Nicoletti, J. A. (2016). Fundamento y construcción del Acto Educativo.

Ord, J. (2016). Curriculum as process: principles of procedure and curriculum as development. In Youth Work Process, Product and Practice (pp. 66-76). Routledge.

Rodríguez-Castro, M. (2018). An integrated curricular design for computer-assisted translation tools: developing technical expertise. The Interpreter and Translator Trainer, 1-20.

Rodríguez, R. R. (2017). Investigación y curriculo. TED: Tecné, Episteme y Didaxis, (6).

Pardasani, M. (2018). Recruiting, engaging, and educating social work leaders: an innovative curricular model. Social Work Education, 37 (4), 519-534.

Peralta, D. A. S., \& Aguja, F. A. P. (2015). Percepción académica sobre la evaluación en el contexto universitario modalidad a Distancia Tradicional y virtual. Cultura Educación y Sociedad, 6(1).

Sánchez, G. Á. (2016). Metodología para el diseño curricular en los Programas Nacionales de Formación (PNF). Espacio Abierto, 24(4).

Stenhouse, L. (2003). Investigación y desarrollo del currículum. Ediciones Morata.

Tobón, S. (2014). Metodología de gestión curricular: una perspectiva socioformativa (No. 378.1 T629c). Trillas.

Tobón, S. (2008). La formación basada en competencias en la educación superior: el enfoque complejo. México: Universidad Autónoma de Guadalajara.

Tobón, S. (2007). El enfoque complejo de las competencias y el diseño curricular por ciclos propedéuticos. Universidad de Los Andes (ULA).

UNIMINUTO (2008). Reglamento Orgánico. Acuerdo No. 090 de diciembre 15 de 2008. Artículo 93 y 94 . Recuperado de:

http://www.uniminuto.edu/documents/941377/941434/A cu+090+Reglamento+Org\%C 3\%A1nico.pdf/99e5f46f-f95a-4b84-89bb-04d1f32d81e2

Universidad Colegio Mayor de Nuestra Señora del Rosario (2004). Lineamientos Institucionales para La Gestión Curricular. Vicerrectoría-Departamento De Planeación Y Desarrollo Académico. Recuperado de http://www.urosario.edu.co/urosario_files/ff/ff409a82-a7894e37-960d-c404cbef1f55.pdf

Universidad de La Salle (2013). Lineamiento para la Gestión Curricular Articulada a la Praxis Investigativa. Colección Librillos Institucionales $\mathrm{N}^{\circ} 56$.

Velasco, M \& Mosquera, F (2008) Estrategias didácticas para el aprendizaje colaborativo. Universidad Distrital Francisco José de Caldas. Santa Fe de Bogotá.

Vincent, S., \& Focht, W. (2011). Interdisciplinary environmental education: elements of field identity and curriculum design. Journal of Environmental Studies and Sciences, 1(1), 14-35.

Zabalza, M. Á. (1997). Diseño y desarrollo curricular (ed. $7^{\circ}$ ). Narcea Ediciones. 